\title{
Accurate Optimization Technique for Phase- Gradient Metasurfaces used in Compact Near-Field Meta-Steering Systems
}

Khushboo Singh ( $\nabla$ khushboo.singh@uts.edu.au )

University of Technology Sydney

Muhammad U. Afzal

University of Technology Sydney

Karu P. Esselle

University of Technology Sydney

\section{Research Article}

Keywords: Near-field Meta-Steering (NFMS), phase-gradient metasurfaces (PGMs), 2D steering system, wireless backhaul mesh networks.

Posted Date: November 30th, 2021

DOI: https://doi.org/10.21203/rs.3.rs-1080116/v1

License: (c) (i) This work is licensed under a Creative Commons Attribution 4.0 International License. Read Full License

Version of Record: A version of this preprint was published at Scientific Reports on March 8th, 2022. See the published version at https://doi.org/10.1038/s41598-022-08143-x. 


\title{
Accurate Optimization Technique for Phase-Gradient Metasurfaces used in Compact Near-Field Meta-Steering Systems
}

\author{
Khushboo Singh ${ }^{1, *}$, Muhammad U. Afzal $^{1}$, and Karu P. Esselle ${ }^{1}$ \\ ${ }^{1}$ University of Technology, School of Electrical and Data Engineering, Sydney, 2007, Australia \\ *khushboo.singh@uts.edu.au
}

\begin{abstract}
Near-field Meta-Steering (NFMS) is a constantly evolving and progressively emerging novel antenna beam-steering technology that involves an elegant assembly of a base antenna and a pair of phase-gradient metasurfaces (PGMs) placed in the near-field region of the antenna aperture. The upper PGM in a Near-Field Meta-Steering system receives an oblique incidence from the lower PGM at all times, a fact that is ignored in the traditional design process of upper metasurfaces. This work proposes an accurate optimization method for metasurfaces in NFMS systems to reduce signal leakage by suppressing the grating lobes and side lobes that are innate artifacts of beam-steering. We detail the design and optimization approach for both upper and lower metasurface. Compared to the conventionally optimized compact 2D steering system, the proposed system exhibits higher directivity and lower sidelobe and grating lobe levels within the entire scanning range. The broadside directivity is $1.4 \mathrm{~dB}$ higher, and the sidelobe level is $4 \mathrm{~dB}$ lower in comparison. The beam-steering patterns for the proposed 2D compact design are experimentally validated, and the measured and predicted results are in excellent concurrence. The versatile compatibility of truncated PGMs with a low gain antenna makes it a compelling technology for wireless backhaul mesh networks and future antenna hardware.
\end{abstract}

\section{Introduction}

The growing hope and hype around the Internet of things (IoT) and constantly increasing demand for ubiquitous coverage with ultra-high-speed data over mobile networks has created a new wave of technological revolution in $5 \mathrm{G}$ cellular communication. The mobile backhaul technology, primarily used to feed massive data to the end-users, connects the network hub to the base stations wirelessly. It is the most cost-effective and versatile solution to connect $5 \mathrm{G}$ base stations to the core network to enable flexible and easy installation of base stations in ad-hoc networks, supporting large crowd gatherings such as concerts and sports events. These backhaul networks depend on the line-of-sight communication between two fixed antenna terminals with point-to-point data transmission ${ }^{1}$. Any error due to antenna misalignment should be avoided to establish a robust link quality in these networks. Thus, a medium/high-gain 2D beam-steering-enabled antenna system with a planar profile is desirable to ease the deployment and cope with the wind-induced movements in the lampposts and other 5G small cell installation sites. These antennas must have the ability to transmit and/or receive in any arbitrary direction within a large conical region.

Conventional mainstream configurations for beam-steering include mechanically moving a fixed beam antenna on a gimbal and electronically steering beams of phased arrays. The mechanical steering solutions are bulky, and a more sophisticated system needs expensive motors to ensure the reliability of vital data links. Active electronically scanned arrays are the most agile front-end systems, but because of the high-cost active radio-frequency (RF) components, their use is limited to high-end applications ${ }^{2,3}$. Due to the limitations associated with traditional beam-steering methods, several unconventional technologies have been investigated, including optical beamformers ${ }^{4}$, liquid crystal-based electronic beam-steering ${ }^{5}$, and metasurface antennas $^{6}$. In a basic comparison, the passive beam-steering antennas (without using active RF components) have certain advantages over active beam-steering antennas. The former is relatively cheaper, has better linearity and scalability, low noise, high power handling capability, broader bandwidth, and low DC power consumption, making them suitable for emerging commercial applications.

Near-field Meta-Steering (NFMS) is a state-of-the-art passive beam-steering antenna technology inspired from the opticalbeam scanning principle that is implemented using Risley prisms ${ }^{7-9}$. The concept was first introduced and demonstrated in $2017^{10}$ and since then has been extended by various research groups ${ }^{1-15}$. The configuration of the classical NFMS system is shown in Fig.1. It consists of medium/high-gain base antennas that can create planar phase fronts on the aperture ${ }^{16}$ and a pair of rotating PGMs placed above the base antenna in its near-field region. The first PGM tilts the antenna beam by introducing a phase shift in the antenna electric near field. The beam-tilt angle is controlled through the gradient of phase shift introduced by 


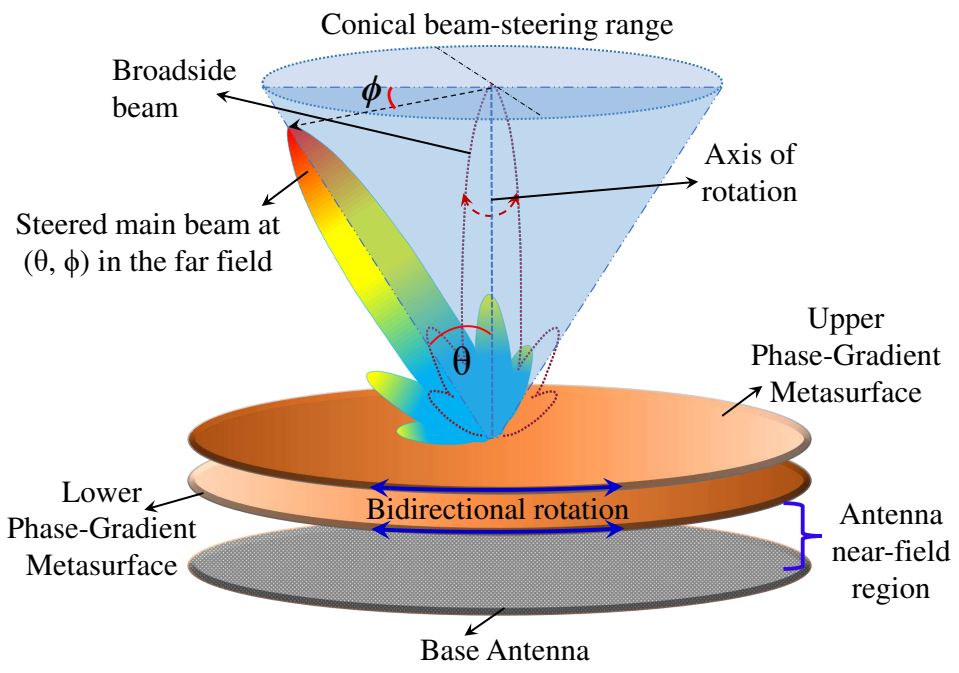

Figure 1. Configuration of classical Near-Field Meta-Steering system.

the PGM. Conventionally, the two PGMs in an NFMS system are identical and introduce the same gradient when placed on the antenna with planar phase fronts. In the NFMS, depending on its orientation, the second PGM can either increase or decrease the effective phase gradient and hence the antenna beam tilt angle. The base antenna remains fixed, and the two PGMs are co/counter-rotated to steer the antenna beam to any location within a wide 2D conical scanning range. The NFMS antenna technology holds several advantages over conventional steering methodologies. The base antenna being entirely stationary obviates the need for RF rotary joints. Since the entire phase shifting is carried out in the near-field region, the entire system is extremely low-profile, making it aesthetically pleasing and easier to be mounted on moving platforms. The PGMs can be rotated with a low-power stepper motor, and the total volume of the system is fixed at all times, even when the beam is steered.

The periodic arrangement of physical cells, referred to as supercells, in PGMs generates periodic grating-type lobes. In addition to these grating lobes, the sidelobes appearing within antenna patterns when the beam is steered to larger elevation angles result in signal leakage, which is a concern for most wireless applications. To ensure efficient steering performance, the PGM pair should be designed strategically. Besides, the sidelobes can be controlled by judiciously tailoring the arrangement of elements in the periodically repeating supercell of the PGMs. The lower metasurface atop the base antenna typically receives a normally incident wave and produces an output beam at an offset angle same as the gradient of the PGM. The design of such PGMs is straightforward and elaborately explained in literature ${ }^{17-20}$. Conventionally, both upper and lower PGMs are designed using the same unit cell-based approach. However, the upper PGM receives an oblique incidence at all times, which calls for a different design and optimization strategy to achieve overall optimal 2D beam-steering performance.

In this work, we propose an innovative and more logical methodology to design and optimize PGMs in an NFMS system by considering the nature of the incident plane wave. The novelty of this work is to design upper PGM with cells having an excellent performance for the obliquely incident electric field. This is the first NFMS design that uses separately characterized cells for the upper and lower PGMs. A Floquet analysis-based CMA-ES optimization similar to the one presented in ${ }^{21}$ has been used to optimize the lower PGM for normally incident plane waves. The upper PGM is optimized assuming obliquely incident plane wave with an angle of incidence same as the phase-gradient of the first PGM. Two compact beam-steering systems are modeled using a medium/low gain RCA as the base antenna. System-I has a pair of conventionally optimized PGMs (optimized for normal plane wave incidence). System-II has lower PGM optimized for normal incidence, and the upper PGM optimized for oblique incidence. The two systems are then compared based on their steering radiation performance. A prototype of the steering system that has better beam-steering performance is fabricated and measured. The results predicted by simulations in CST MWS are experimentally validated.

\section{Design Methodology}

The NFMS system can be operated in three steering modes ${ }^{10}$. One of the modes, where beam is steered in both elevation and azimuth plane is achieved by fixing lower PGM in addition to the base antenna and only rotating the upper PGM. The optimization strategy proposed here, however, can also be implemented without any changes, for other steering modes where both metasurfaces are simultaneously rotated . 


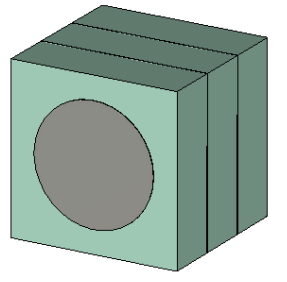

(a)

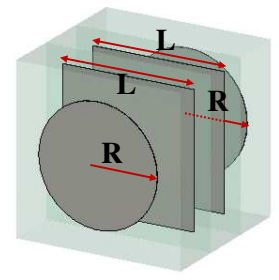

(b)

Figure 2. Phase-transforming cell configuration. (a) Perspective view. (b) Internal configuration of PTC, showing metal patches on four layers.

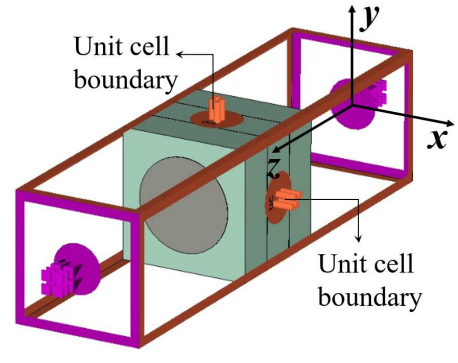

(a)

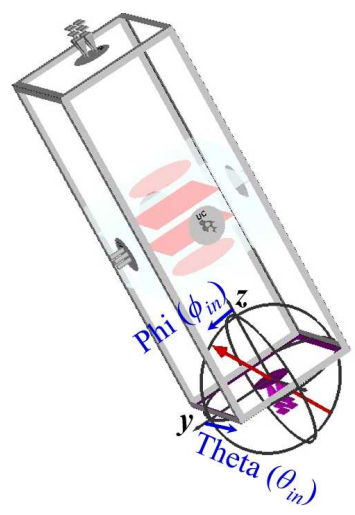

(b)

Figure 3. Simulation set-up for a PTC for the upper PGM in a NFMS system. (a) Perspective view of a square metal patch PTC with unit cell boundary conditions and (b) oblique incidence plane wave direction defined by $\theta_{\text {in }}$ and $\phi_{i n}$.

\section{Supercell Design Approach}

The PGMs are composed of periodic supercells designed using phase-transforming cells (PTCs) arranged in a pre-defined sequence. The lower PGM is designed to tilt a normally incident plane wave at its input to a $30^{\circ}$ angle at the output. The upper PGM is designed to transform this obliquely incident plane back to the normal at its output. The configuration of PTCs used in this work is shown in Fig 2. Each PTC comprises four metal layers (circular patches on top and bottom layer and square patches in the two middle layers) and three dielectric layers of Taconic TLY-5 $\left(\varepsilon_{r}=2.2\right.$, thickness $\left.t=1.5 \mathrm{~mm}\right)$. The side length of PTC is $d=\lambda_{\circ} / 3$ ( $\lambda_{\circ}$ is the free space wavelength), which is equal to $5 \mathrm{~mm} @ 20 \mathrm{GHz}$. For upper PGM, the PTCs are simulated with periodic boundary conditions and oblique incidence excitation, as shown in Fig. 3. The unit cell boundary conditions are applied along $x$ - and $y$-directions. The PTC is excited with an obliquely incident plane wave. The oblique-incident wave propagation vector shown by the red arrow in Fig. 3(b) is defined in terms of $\theta_{i n}$ and $\phi_{i n}$, where $\theta_{i n}$ is the oblique elevation angle, and $\phi_{i n}$ is the azimuth angle. Here, $\theta_{i n}$ is equal to the beam tilt angle of the lower PGM, while $\phi_{i n}$ is kept as zero. To accurately choose the constituent elements for the design of upper PGMs, a parameter sweep is performed on the dimensions of metallic patches in the PTCs. The values of transmission magnitudes and corresponding transmission phases are stored in database-I. For lower PGM, the unit cell is excited with a normally incident plane wave and simulated with periodic boundary conditions. The design parameters for lower PGM are then obtained by performing a parameter sweep on the dimensions of metallic patches in the PTCs. The values of transmission magnitudes and corresponding transmission phases are stored in a database-II.

In a PGM, each periodically repeating supercell cover the phase range from $0^{\circ}$ to $360^{\circ}$. For a $30^{\circ}$ beam-tilt, a phase shift $\Delta \phi=60^{\circ}$ between adjacent cell in a supercell is calculated from the equation below: ${ }^{22,23}$

$$
\Delta \phi=\frac{2 \pi}{\lambda_{\circ}} d \sin \theta,
$$

where $d$ is the size of the cell in terms of free-space wavelength. The number of unique PTCs $n=2 \pi / \Delta \phi=6$. Hence, we select six different unit cells with high transmission and desired transmission phases from database-I to design supercells for upper PGM. The other six desired unit cells were selected from database-II to design supercells for lower PGM. The dimension 
Table 1. Dimensions of metallic patches in the supercell of upper PGM and lower PGM

\begin{tabular}{|ll|ll|}
\hline \multicolumn{2}{|c|}{ Upper PGM Supercell } & \multicolumn{2}{c|}{ Lower PGM supercell } \\
\hline$L_{n}(\mathrm{~mm})$ & $R_{n}(\mathrm{~mm})$ & $L_{n}(\mathrm{~mm})$ & $R_{n}(\mathrm{~mm})$ \\
\hline \hline$L_{1}=3.47$ & $R_{1}=2$ & $L_{1}=3.8$ & $R_{1}=1.95$ \\
$L_{2}=3.8$ & $R_{2}=2.15$ & $L_{2}=3.9$ & $R_{2}=2.15$ \\
$L_{3}=4.13$ & $R_{3}=2.24$ & $L_{3}=4$ & $R_{3}=2.25$ \\
$L_{4}=0.1$ & $R_{4}=0.058$ & $L_{4}=0.1$ & $R_{4}=0.05$ \\
$L_{5}=2.96$ & $R_{5}=0.98$ & $L_{5}=3$ & $R_{5}=0.95$ \\
$L_{6}=3.29$ & $R_{6}=1.91$ & $L_{6}=3.3$ & $R_{6}=1.9$ \\
\hline
\end{tabular}

of metallic patches in the supercell of upper and lower PGM is provided in Table 1 . Usually, the phase is wrapped back to $0^{\circ}$ at the edge of the supercell, and this pattern repeats. The EM simulation tool (CST MWS) uses periodic boundary conditions to simulate the periodicity of the supercells. For our analysis we shifted the phase-wrapping point from edges to the middle of the supercell. Fig. 4 shows two supercells, one with phase wrapping on the edges and the other with the phase wrapping in the

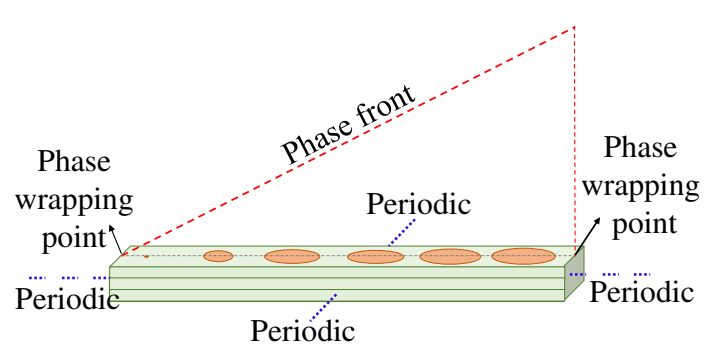

(a)

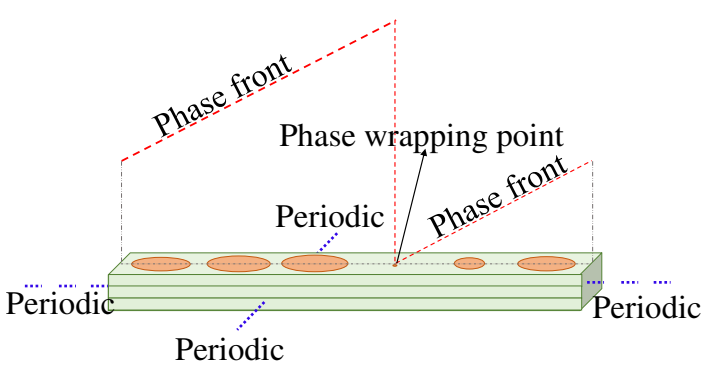

(b)

Figure 4. Supercells for $30^{\circ}$ beam-tilt with (a) phase wrapping at the edges, (b) with phase wrapping somewhere in the middle.

middle. It should be noted that a shift in the absolute phase values does not affect the overall performance of the metasurfaces as long as the relative phase difference between the adjacent cells is kept the same.

\section{Optimization of PGMs in NFMS system}

Optimization of PGMs using a Floquet analysis-based approach on periodic supercells has been elaborately discussed in ${ }^{21}$. Here, a similar approach is used to optimize the lower PGM. However, the upper PGM is optimized with a different simulation setup considering the oblique nature of the incidence wave. The dimensions of the circular and square metal patches are optimized using the CMA-ES algorithm in the CST optimizer. Since the optimization variables are continuous, the optimization algorithms inbuilt in CST work reasonably well. The initial dimensions from Table 1 are used as a seed for the algorithm in both upper and lower PGM optimization.

The supercell for lower PGM is excited with a plane wave incident normally from input Floquet port. In contrast, the supercell for upper PGM is excited with an obliquely incident TE-polarized plane wave originating from the input Floquet port. The orientation of oblique incidence is defined in terms of elevation angle $\theta_{i n}$, which is equal to the beam tilt obtained from the bottom PGM and azimuth angle $\phi_{i n}=180^{\circ}$. This simulation setup mimics the orientation of PGM-pair in an NFMS system when $\psi_{1}=0^{\circ}, \psi_{2}=180^{\circ}\left(\psi_{1}-\psi_{2}=180^{\circ}\right)$ and the beam is in the broadside direction. The supercells support 10 propagating modes, each. The optimization goal is to bring 9 undesired modes (UDMs) below $-35 \mathrm{~dB}$ and simultaneously maintain the desired mode (DM) above $-0.1 \mathrm{~dB}$. The fitness function (FF) in equation (2) is defined as a weighted sum of objectives mentioned above to selectively suppress the unwanted grating lobes:

$$
F F=\left[w_{m}\{\max (0,(-0.1-D M))\}\right]^{2}+\sum_{i=1}^{9}\left[w_{i}\{\max (0,(U D M-(-35)))\}\right]^{2},
$$

where $w_{m}$ is the weight associated with the desired mode $(D M)$, and $w_{i}$ are the weights associated with the undesired modes $(U D M)$. The value of $w_{m}$ is fixed to 20 , and $w_{i}$ can vary between 1 to 19 . To formulate a single fitness function that ensures high transmission of desired mode while simultaneously suppresses the transmission of spurious grating lobes, the maximum 
Table 2. Dimensions of supercells and grating lobe levels before and after optimization $(\mathrm{mm})$

\begin{tabular}{|c|c|c|c|c|c|c|}
\hline Supercells & \multicolumn{2}{|c|}{$\begin{array}{l}\text { Initial Dimensions } \\
(\mathrm{mm})\end{array}$} & \multicolumn{2}{|c|}{$\begin{array}{l}\text { Optimized Dimensions } \\
(\mathrm{mm})\end{array}$} & $\begin{array}{c}\text { Grating lobe level } \\
\text { before optimization }(\mathrm{dB})\end{array}$ & $\begin{array}{c}\text { Grating lobe level } \\
\text { after optimization }(\mathrm{dB})\end{array}$ \\
\hline $\begin{array}{l}\text { Lower } \\
\text { PGM }\end{array}$ & $\begin{array}{l}\text { L1 }=3.8 \\
\text { L2 }=3.9 \\
\text { L3 }=4 . \\
\text { L4 }=0.1 \\
\text { L5 }=3 \\
\text { L6 }=3.4\end{array}$ & $\begin{array}{l}\mathrm{R} 1=1.9 \\
\mathrm{R} 2=2.15 \\
\mathrm{R} 3=2.15 \\
\mathrm{R} 4=0.05 \\
\mathrm{R} 5=0.95 \\
\mathrm{R} 6=1.9\end{array}$ & $\begin{array}{l}\mathrm{L} 1=3.44 \\
\mathrm{~L} 2=3.91 \\
\mathrm{~L} 3=4.08 \\
\mathrm{~L} 4=0.09 \\
\mathrm{~L} 5=3.05 \\
\mathrm{~L} 6=3.28\end{array}$ & $\begin{array}{l}\mathrm{R} 1=1.95 \\
\mathrm{R} 2=2.13 \\
\mathrm{R} 3=2.23 \\
\mathrm{R} 4=0.05 \\
\mathrm{R} 5=0.98 \\
\mathrm{R} 6=1.93\end{array}$ & $\begin{array}{l}T_{0}=-13.5 \mathrm{~dB}\left(0^{\circ}\right) \\
T_{-1}=-14.01 \mathrm{~dB}\left(-30^{\circ}\right) \\
T_{+1}=-0.59 \mathrm{~dB}\left(30^{\circ}\right) \\
T_{-2}=-28.18 \mathrm{~dB}\left(-60^{\circ}\right) \\
T_{+2}=-22.36 \mathrm{~dB}\left(60^{\circ}\right)\end{array}$ & $\begin{array}{l}T_{0}=-30.57 \mathrm{~dB}\left(0^{\circ}\right) \\
T_{+1}=-0.13 \mathrm{~dB}\left(30^{\circ}\right) \\
T_{-1}=-30.07 \mathrm{~dB}\left(-30^{\circ}\right) \\
T_{+2}=-29.9 \mathrm{~dB}\left(60^{\circ}\right) \\
T_{-2}=-31.65 \mathrm{~dB}\left(-60^{\circ}\right)\end{array}$ \\
\hline $\begin{array}{l}\text { Upper } \\
\text { PGM }\end{array}$ & $\begin{array}{l}\text { L1 }=3.47 \\
\text { L2 }=3.8 \\
\text { L3 }=4.13 \\
\text { L4 }=0.1 \\
\text { L5 }=2.96 \\
\text { L6 }=3.29\end{array}$ & $\begin{array}{l}\mathrm{R} 1=2 \\
\mathrm{R} 2=2.15 \\
\mathrm{R} 3=2.24 \\
\mathrm{R} 4=0.06 \\
\mathrm{R} 5=0.98 \\
\mathrm{R} 6=1.91\end{array}$ & $\begin{array}{l}\mathrm{L} 1=3.27 \\
\mathrm{~L} 2=3.93 \\
\mathrm{~L} 3=4.11 \\
\mathrm{~L} 4=0.1 \\
\mathrm{~L} 5=3.07 \\
\mathrm{~L} 6=2.93\end{array}$ & $\begin{array}{l}\mathrm{R} 1=2.12 \\
\mathrm{R} 2=2.1 \\
\mathrm{R} 3=2.18 \\
\mathrm{R} 4=0.04 \\
\mathrm{R} 5=0.87 \\
\mathrm{R} 6=1.93\end{array}$ & $\begin{array}{l}T_{0}=-0.63 \mathrm{~dB}\left(0^{\circ}\right) \\
T_{-1}=-14.16 \mathrm{~dB}\left(-30^{\circ}\right) \\
T_{+1}=-12.43 \mathrm{~dB}\left(30^{\circ}\right) \\
T_{-2}=-32.49 \mathrm{~dB}\left(-60^{\circ}\right) \\
T_{+2}=-28.09 \mathrm{~dB}\left(60^{\circ}\right)\end{array}$ & $\begin{array}{l}T_{0}=-0.169 \mathrm{~dB}\left(\theta=0^{\circ}\right) \\
T_{-1}=-36.74 \mathrm{~dB}\left(-30^{\circ}\right) \\
T_{+1}=-30.11 \mathrm{~dB}\left(30^{\circ}\right) \\
T_{+2}=-32.15 \mathrm{~dB}\left(60^{\circ}\right) \\
T_{-2}=-30.35 \mathrm{~dB}\left(-60^{\circ}\right)\end{array}$ \\
\hline
\end{tabular}

weight is assigned to increase the desired mode above $-0.1 \mathrm{~dB}$ and the undesired modes are assigned weights proportional to their magnitude in the initial design to reduce them below $-35 \mathrm{~dB}$. The weights assigned to undesired modes must never be greater than that assigned to the desired mode since the primary objective is to have maximum energy directed to the desired mode and only then ensure suppression in undesired modes. The weights are higher for the UDMs with higher magnitude and vice-versa.

The CMA-ES algorithm minimizes the fitness function defined in equation (2) in both lower and upper PGM optimization. The algorithm was stopped manually when there was no improvement (reduction) in fitness function for last 50 runs. The simulation time for the supercell is approximately 7 minutes and 21 seconds. The upper supercell optimization was stopped after 684 function evaluations. The lower supercell completed 853 function evaluations before it was terminated manually. The dimensions of the initial and optimized supercell for both upper and lower PGMs are provided in Table 2. The magnitude of grating lobes before and after optimization are also provided in Table 2, for both upper and lower PGMs. CST array calculator is used to generate the far-field pattern for a metasurface aperture of $\left(12 \lambda_{0} \times 12 \lambda_{0}\right)$ designed by repeating the two supercells six times along $x$-direction and 36 times along $y$-direction. The predicted radiation pattern for initial and optimized lower PGM is compared in Fig 5(a). Fig. 5(b) compares the predicted broadside radiation patterns of initial and optimized upper PGMs. We

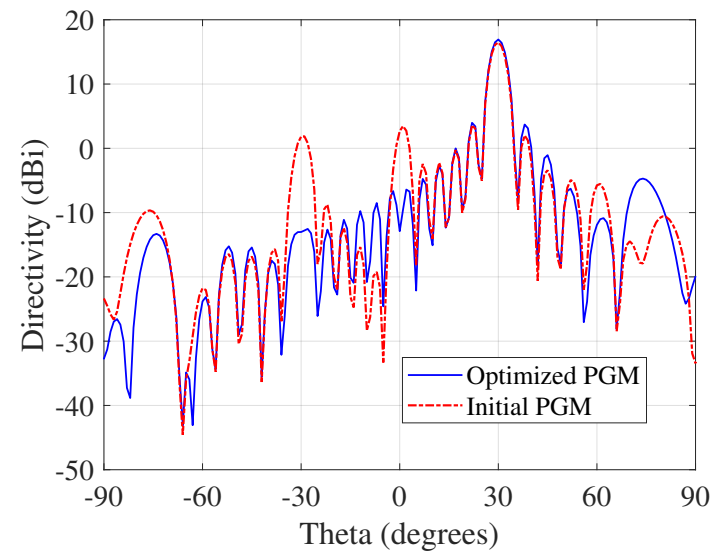

(a)

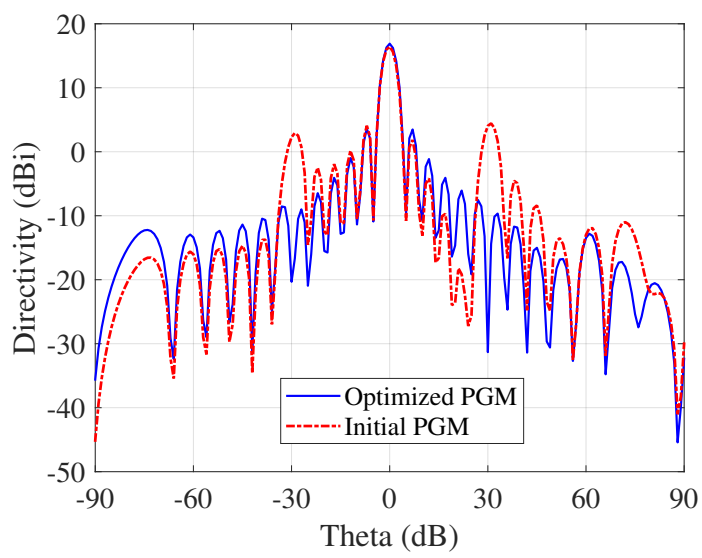

(b)

Figure 5. (a) Comparison between the steered radiation pattern predicted using CST array calculator for $12 \lambda_{0} \times 12 \lambda_{0}$ aperture of optimized lower PGM and initial lower PGM when excited with a normally incident plane wave. (b) Comparison between the steered radiation pattern predicted using CST array calculator for $12 \lambda_{0} \times 12 \lambda_{0}$ aperture of optimized lower PGM and initial lower PGM when excited with an oblique incidence $\left(\theta_{i n}=30^{\circ}\right.$ and $\left.\phi_{i n}=180^{\circ}\right)$.

observe that the grating lobes in the predicted steered radiation pattern of lower PGM and the broadside radiation pattern of 


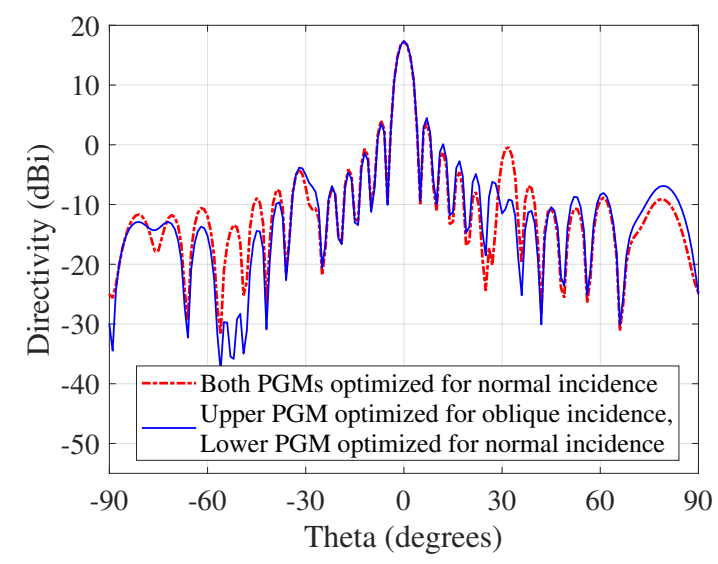

Figure 6. Comparison between the broadside radiation pattern predicted using CST array calculator for a pair of conventionally optimized PGMs for normal incidence and a pair of PGMs where the upper PGM is optimized for oblique incidence while lower PGM is optimized for normal incidence. The PGMs pairs are aligned such that $\psi_{1}=0^{\circ}$ and $\psi_{1}=180^{\circ}$.

upper PGM are higher for the initial unoptimized supercell than the optimized supercell. However, the above simulation setups assumes an ideal scenario when the incident plane wave is entirely uniform. In practice, the PGMs exists in pair, placed above (and close to) the base antenna, in a NFMS system. To study the actual performance, the supercell of upper PGM optimized for oblique incidence was rotated $180^{\circ}$ and placed above the supercell of lower PGM optimized conventionally (assuming normal incidence) at a distance of $\lambda_{0} / / 4$ such that it represents the PGM-pair orientation when $\psi_{1}=0$ and $\psi_{2}=180^{\circ}$. The setup was excited with a normal plane wave incidence. The broadside radiation pattern predicted using the CST array calculator is compared with the pattern of a similarly arranged pair of conventionally optimized supercells (assuming normal incidence) in Fig. 6. In this way, we get a more accurate prediction of the radiation pattern for the full PGM-pair when placed in the near field of a high gain antenna with uniform plane wave output. It is observed that the radiation pattern for a PGM-pair has lower grating lobes when upper PGM is optimized for oblique incidence and lower PGM is optimized for normal incidence. It is imperative to optimize the PGMs in an NFMS system strategically to enhance overall system performance.

\section{Design Example}

We demonstrated the complete system by developing a compact NFMS system. The base antenna is a resonant cavity antennas (RCAs), which is also referred to as leaky-wave antenna, Fabry-Perot antenna and electromagnetic bandgap (EBG) antenna in the literature ${ }^{24,25}$. RCAs are simple, efficient, and planar antennas with high directivity. They can be designed for both liner and circular polarizations. We uses a compact, strongly truncated RCA as a source of EM radiation that is known for its planar phase fronts. The base antenna is linearly polarized with the electric field parallel to the y-axis.

\section{Compact Beam-Steering RCA System Design}

The configuration of the compact NFMS system with three-dimensional model is shown in Fig. 7. It has a classical RCA having uniform dielectric superstrate made of circular disc of Rogers TMM 10. The superstrate is placed at half-wavelength

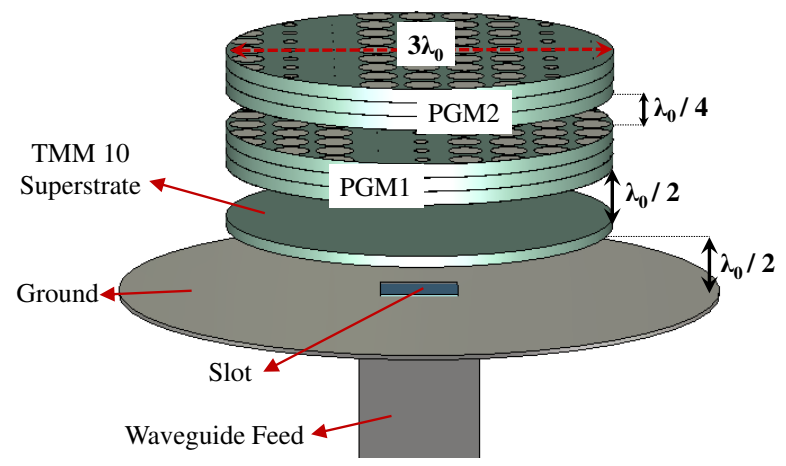

Figure 7. Compact NFMS system design. 
$\left(\lambda_{0} / 2\right)$ above a circular metallic ground plane that has a slot in the middle. This cavity formed between ground and superstrate is excited through the slot (of size $6 \mathrm{~mm} \times 6 \mathrm{~mm}$ ) with a rectangular waveguide at the operating frequency of $20 \mathrm{GHz}$. The diameter of the ground plane is not due to electromagnetic design requirement but only to securely mount the antenna on the AUT stage in the anechoic chamber. The superstrate's diameter is kept as $3 \lambda_{0}$, where $\lambda_{0}$ is the free space wavelength at $20 \mathrm{GHz}$. The relative permittivity of Rogers TMM 10 dielectric superstrate is $\varepsilon_{r}=9.8$. The thickness of the superstrate is $t=0.25 \lambda_{g}=0.25 \times \lambda_{0} / \sqrt{\varepsilon_{r}}$ at $20 \mathrm{GHz}$ operating frequency. The directivity of the RCA increases with the increase in the permittivity of the superstrate due to an increase in the reflectivity. The boresight directivity of this classical single layer type RCA is approximately $17 \mathrm{dBi}$. A pair of truncated PGMs with an aperture of diameter $3 \lambda_{0}$ (same as the RCA superstrate) is placed half wavelength above the RCA aperture. The RCA produces an electric field with a fairly uniform phase in the near-field, which creates a broadside beam in the far-field. The relative rotation between the PGM-pair causes the beam to steer in the 3D conical volume/space and the complete steering process is explained in brief in ${ }^{21}$ and in detail in ${ }^{10}$. When the two identical PGMs are aligned such that $\psi_{1}=0^{\circ}$ and $\psi_{2}=180^{\circ}$, where $\psi_{1}$ and $\psi_{2}$ are the orientation angles of the two PGMs, the beam is in the broadside direction. If the lower metasurface is fixed at $\psi_{1}=0$ and $\psi_{2}$ is varied from $180^{\circ}$ to $0^{\circ}$, the azimuth angle decreases according to $\phi=\psi / 2$, while the elevation angle gradually increases to $\theta_{\max }$ for a certain value of $\psi_{2}$ (can be calculated using of phase method in ${ }^{26}$ ), beyond which the beam goes in the lower invisible hemisphere until $\psi_{2}=0^{\circ}$.

\section{Results}

We designed two compact beam-steering systems of same dimensions as specified in Fig. 7, namely, System-I and System-II. System-I is composed of compact RCA and a pair of PGMs optimized conventionally (considering normal incidence). System-II comprises of compact RCA and a pair of optimized PGMs where upper PGM is optimized for oblique incidence and lower PGM is optimized for normal incidence.

\section{Comparing two Compact Beam-Steering Systems}

The elevation pattern cuts for System-I and System-II are compared in Fig. 8 for several orientations of upper PGM when lower PGM is fixed at $\psi_{1}=0^{\circ}$. For System-I, when the beam is steered away from broadside $\left(\Theta=0^{\circ}\right)$ to elevation angles $13^{\circ}$,

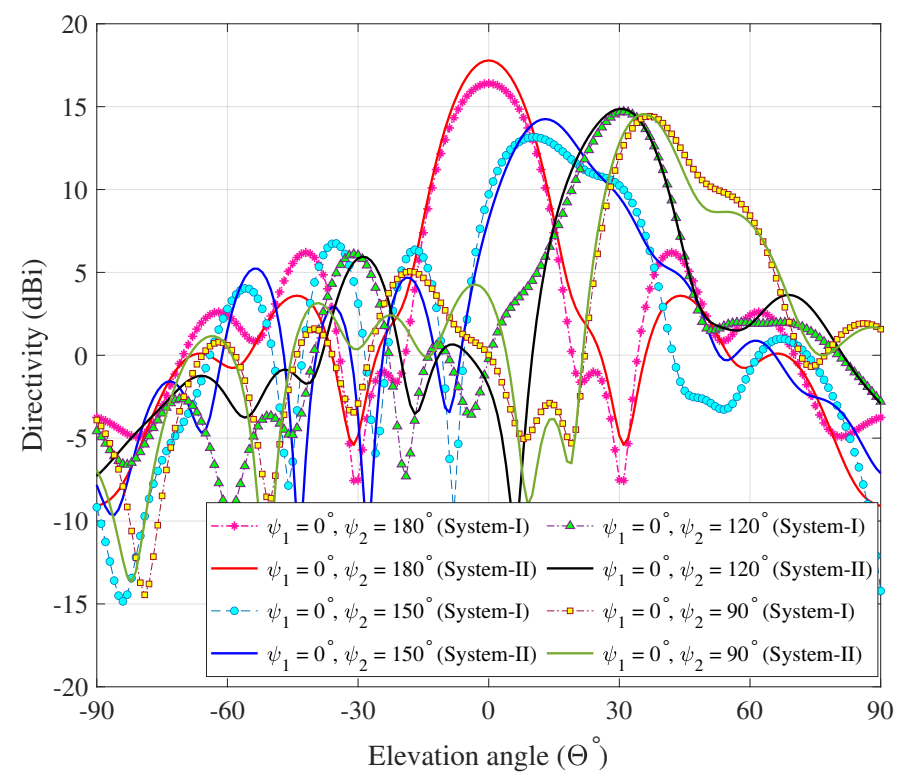

Figure 8. Elevation plane radiation pattern cuts for each rotation of MS2 ( $\psi_{1}=0$ and $\psi_{2}$ is varied $)$ in the compact NFMS system.

$30^{\circ}$ and $35^{\circ}$, the directivity changes from $16.4 \mathrm{dBi}$, to $13.2 \mathrm{dBi}, 14.7 \mathrm{dBi}$ and $14.4 \mathrm{dBi}$ and the side-lobe level changes from $-10.2 \mathrm{~dB}$ to $-6.4 \mathrm{~dB},-8.6 \mathrm{~dB}$ and $-9.1 \mathrm{~dB}$, respectively. For System-II, the directivity changes from $17.8 \mathrm{dBi}$, to $14.3 \mathrm{dBi}$, $14.9 \mathrm{dBi}$, and $15 \mathrm{dBi}$, and the side-lobe level changes from $-14.2 \mathrm{~dB}$ to $-9.1 \mathrm{~dB},-8.9 \mathrm{~dB}$, and $-12.3 \mathrm{~dB}$, when the beam is steered to elevation angles $13^{\circ}, 30^{\circ}$ and $35^{\circ}$, respectively. We observe that System-II has higher directivity and lower SLL for all beam locations, hence we select System-II for further study and final prototype. We then characterize System-II based on steering angle, sidelobes, and directivity. Fig. 9 shows 1D polar plots of radiation patterns for four different orientations (corresponding to four different beam locations) for System-II. Fig 10 shows the 3D patterns for the same four orientations. 
Farfield Directivity Abs (Phi=90)

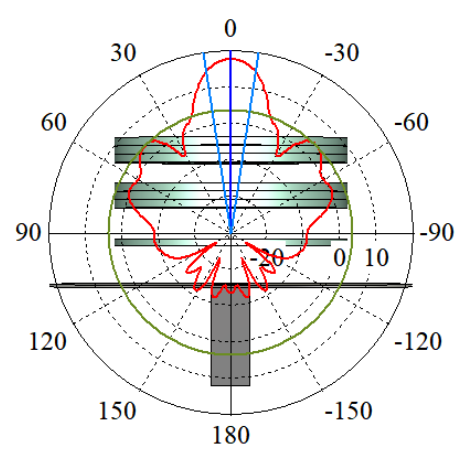

Theta / Degree vs. dBi (a)
Frequency $=20 \mathrm{GHz}$

Main lobe magnitude $=17.8 \mathrm{dBi}$

Main lobe direction $=0.0 \mathrm{deg}$. Angular width $(3 \mathrm{~dB})=17.2 \mathrm{deg}$. Side lobe level $=-14.2 \mathrm{~dB}$

Farfield Directivity Abs $(\mathrm{Phi}=60)$

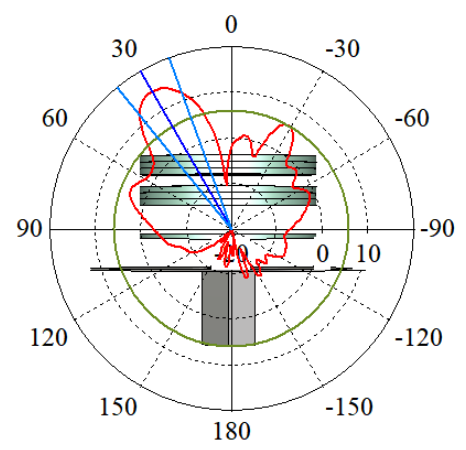

Theta / Degree vs. dBi

(c)

Farfield Directivity Abs (Phi=76)

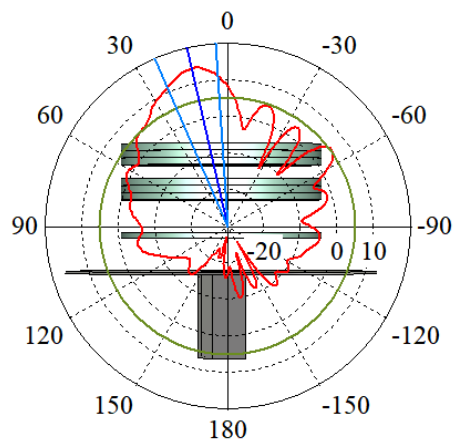

Theta / Degree vs. dBi

Frequency $=20 \mathrm{GHz}$

Main lobe magnitude $=14.2 \mathrm{dBi}$

Main lobe direction $=13.0 \mathrm{deg}$.

Angular width $(3 \mathrm{~dB})=19.8 \mathrm{deg}$.

Side lobe level $=-9.1 \mathrm{~dB}$ (b)

Farfield Directivity Abs (Phi=48)

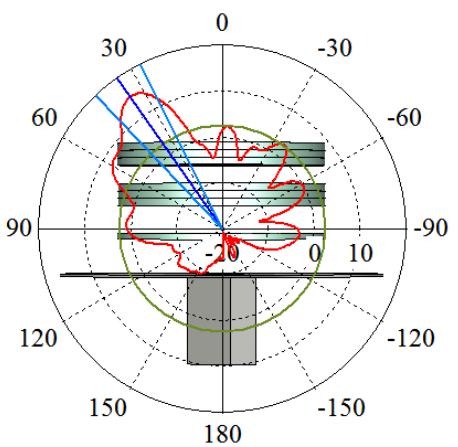

Theta / Degree vs. dBi
Frequency $=20 \mathrm{GHz}$

Main lobe magnitude $=15 \mathrm{dBi}$ Main lobe direction $=35.0 \mathrm{deg}$. Angular width $(3 \mathrm{~dB})=16.6 \mathrm{deg}$. Side lobe level $=-12.3 \mathrm{~dB}$
Main lobe direction $=30.0 \mathrm{deg}$.
Angular width $(3 \mathrm{~dB})=18.8 \mathrm{deg}$. Side lobe level $=-8.9 \mathrm{~dB}$

(d)

Figure 9. 1D polar plots for three different orientations of upper PGM when lower PGM is fixed $\left(\psi_{1}=0\right)$. (a) $\psi_{2}=180^{\circ}$ (b) $\psi_{2}=150^{\circ}$ (c) $\psi_{2}=120^{\circ}$ and (d) $\psi_{2}=90^{\circ}$.

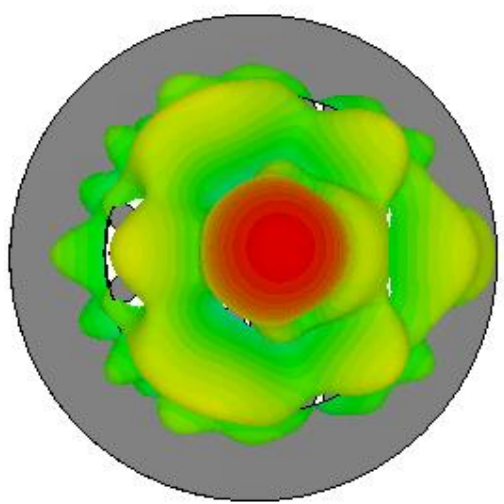

(a)
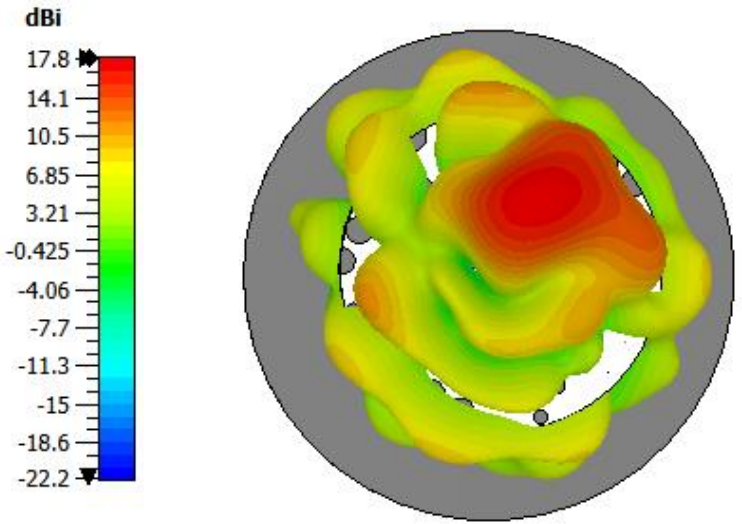

(b)

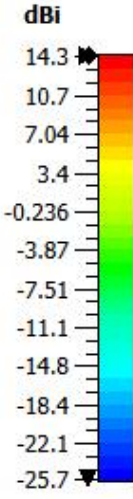

Figure 10. 3D radiation pattern for compact NFMS System (front view) when $\psi_{1}=0^{\circ}$ at all times and $\psi_{2}$ is varied: (a) $\psi_{2}=180^{\circ}$, (b) $\psi_{2}=135^{\circ}$ 


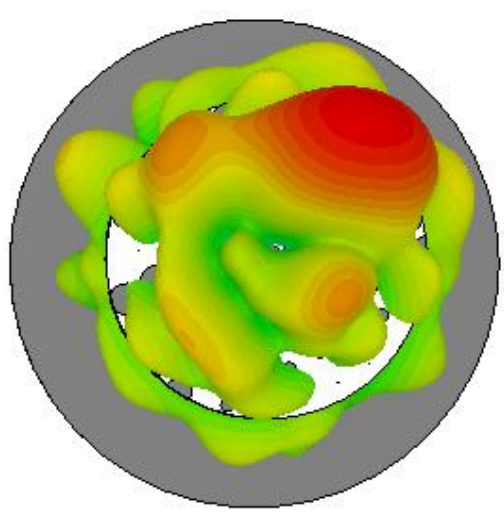

(c)
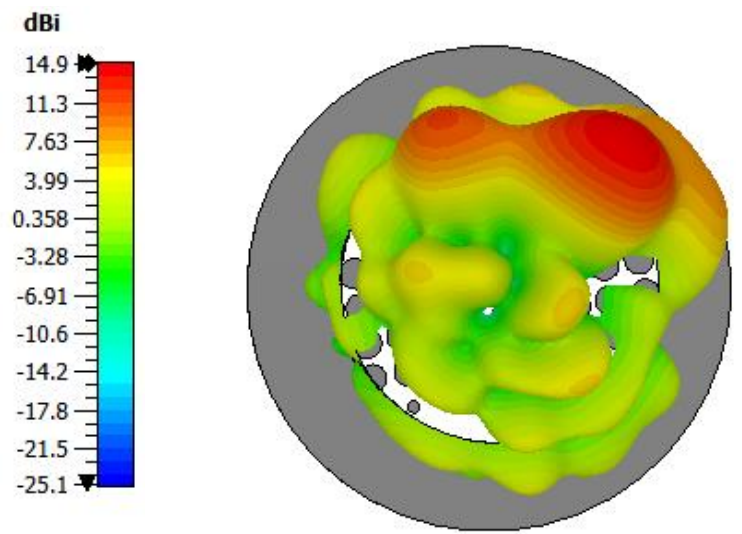

(d)
dBi

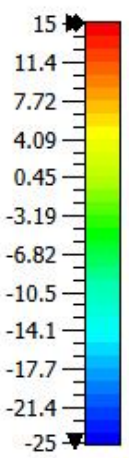

Figure 10. 3D radiation pattern for compact NFMS System (front view) when $\psi_{1}=0^{\circ}$ at all times and $\psi_{2}$ is varied: (c) $\psi_{2}=120^{\circ}$ and (d) $\psi_{2}=90^{\circ}$.

\section{Fabricated Prototype}

The prototypes for the PGM pair of System-II along with the single-layer uniform-superstrate RCA were fabricated as shown in Fig. 11. The fabricated components are assembled into compact near-field meta-steering system whose steering performance is

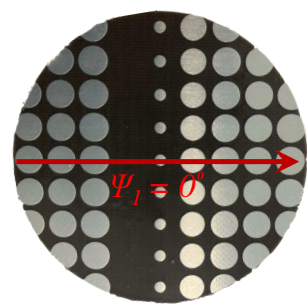

(a)

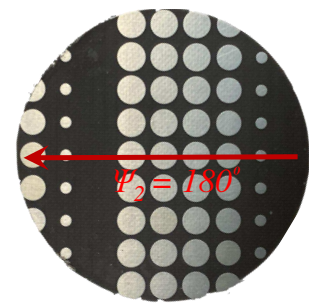

(b)

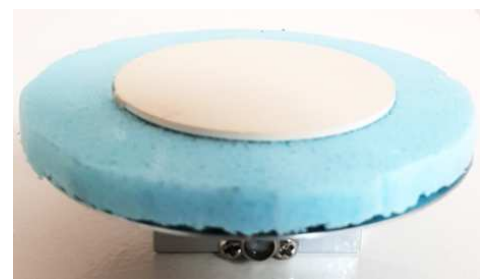

(c)

Figure 11. Fabricated prototype of (a) Lower phase-gradient metasurface, (b) Upper phase-gradient metasurface, (c) Single-layer uniform-superstrate resonant cavity antenna. The diameter of both metasurfaces and RCA superstrate is $3 \lambda_{0}$.

measured for several configurations to validate numerically predicted results. The primary objective of the proposed design methodology is to demonstrate the steering of antenna beam in both azimuth and elevation planes, with reduced side-lobes and grating lobe levels and simultaneously maintain a fairly stable directivity.

\section{Experimental Results with one PGM}

The first antenna assembly comprises of lower PGM and the RCA as shown in Fig. 12. The PGM is placed half a wavelength (7.5 mm @ 20GHz) above the RCA that acts as an electromagnetic illuminator with a nearly uniform phase distribution in the

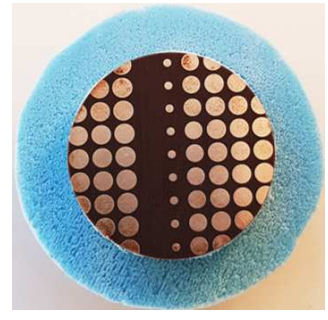

(a)

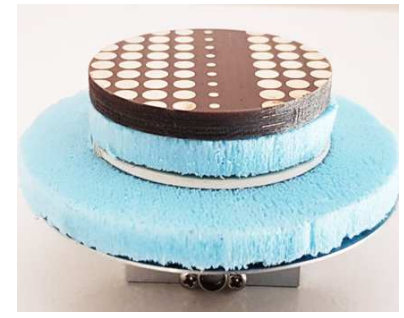

(b)

Figure 12. First antenna measurement assembly of RCA and lower PGM (a) Top view and (b) side view. 


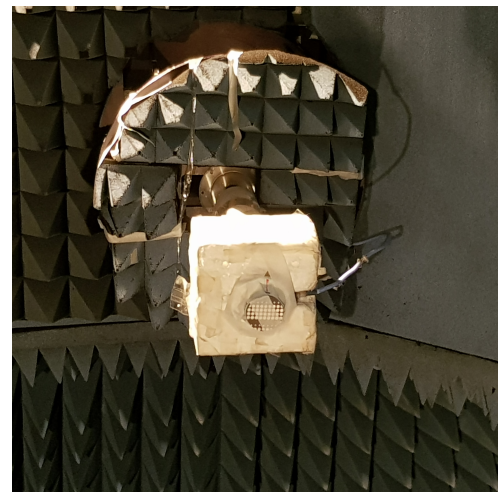

(a)

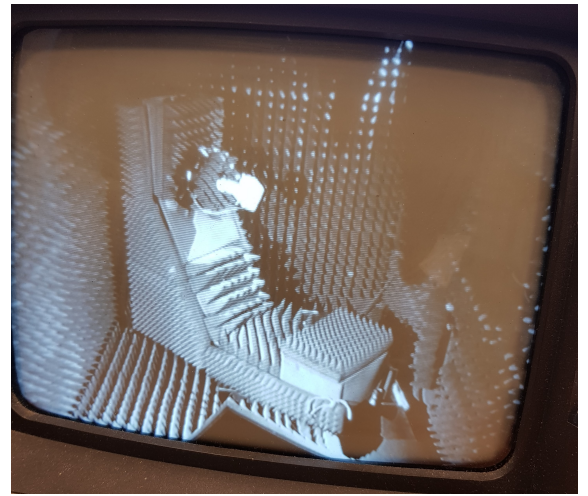

(b)

Figure 13. Photos taken inside the anechoic chamber at Australian Antenna Measurement Facility (a) Device under test setup (b) Monitoring the progress of measurement on a computer screen.

aperture. Far-field radiation pattern was measured in an NSI spherical near-field range at Australian Antenna Measurement Facility. The antenna measurement setup in the anechoic chamber is shown in Fig. 13. The normalized measured radiation pattern of RCA with lower PGM oriented at $\psi_{1}=0^{\circ}$, is compared with the normalized simulated pattern in Fig. 14. The predicted beam-tilt achieved with an assembly of RCA and lower PGM is in close agreement the measured results. The maximum predicted directivity is $14 \mathrm{dBi}$.

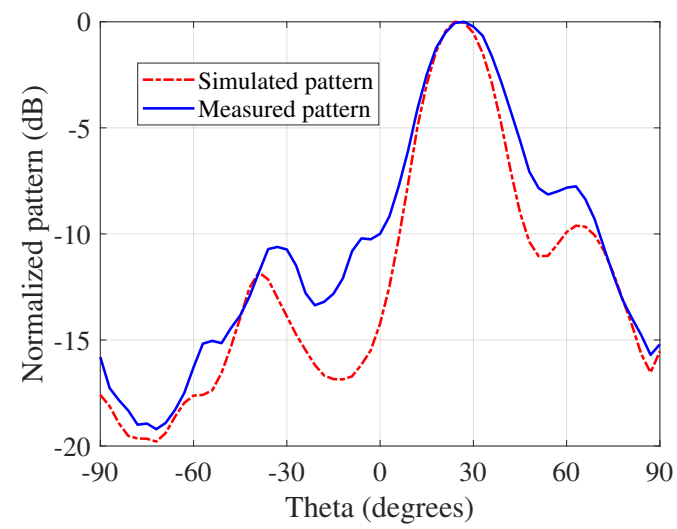

Figure 14. Measured and predicted elevation plane pattern cuts for an assembly of RCA and one PGM.

\section{Experimental Results with PGM-pair}

A photograph of the complete system is shown in Fig. 15. The gap between the two PGMs is quarter wavelength (3.75 mm @

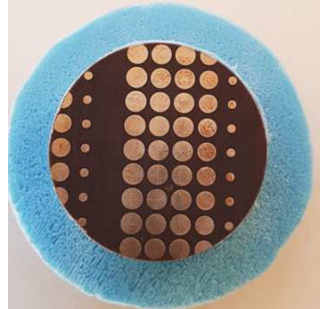

(a)

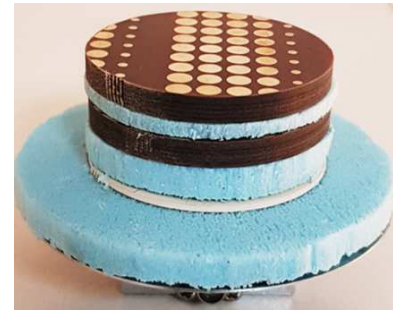

(b)

Figure 15. Second antenna measurement assembly of RCA and lower PGM (a) Top view and (b) side view. 


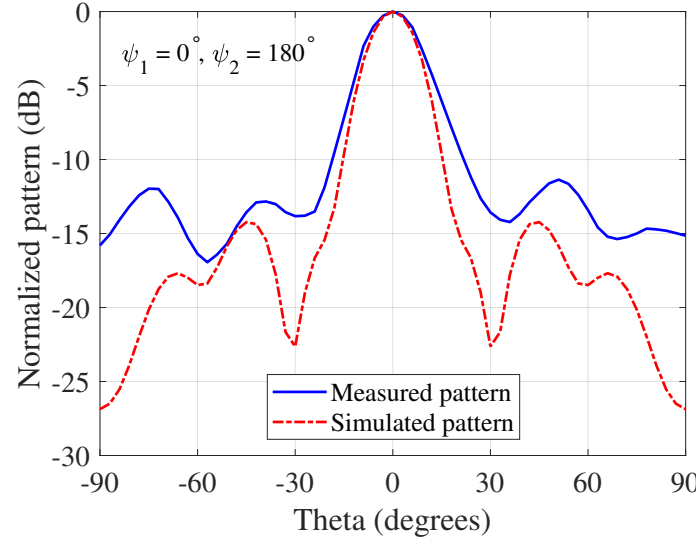

(a)

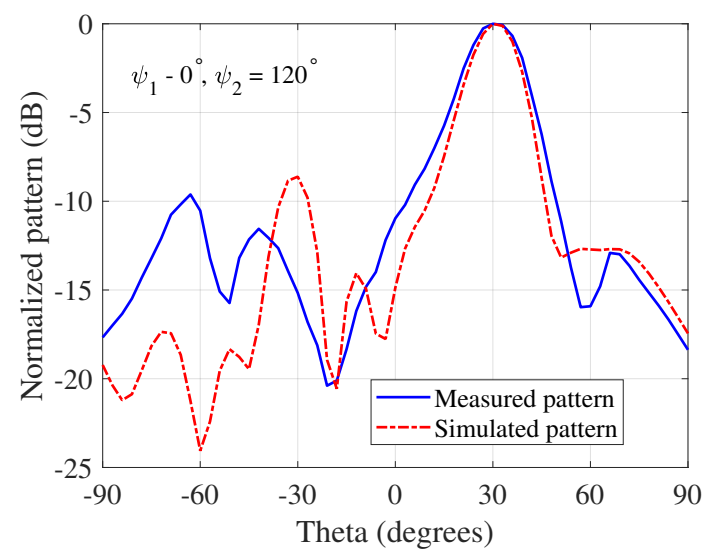

(c)

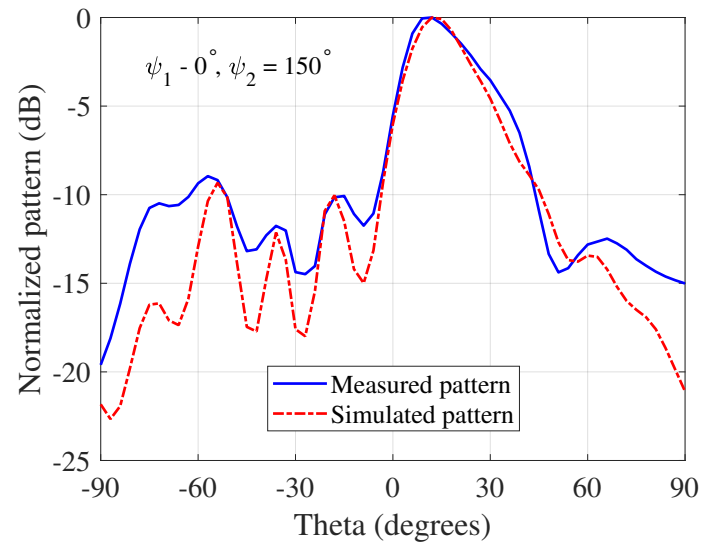

(b)

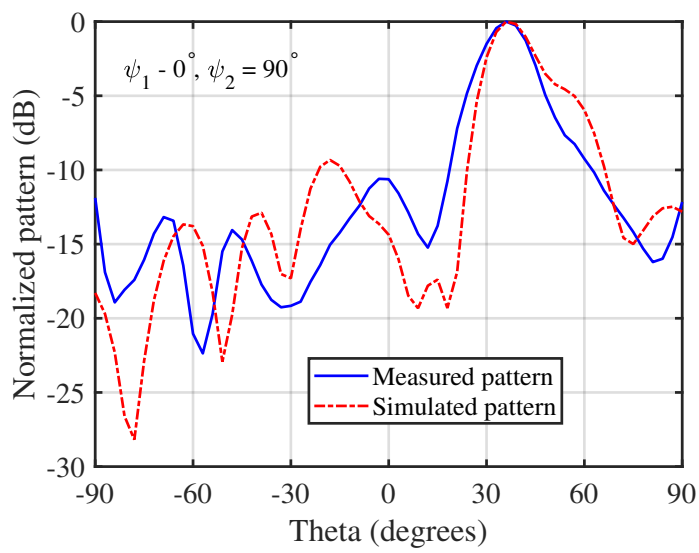

(d)

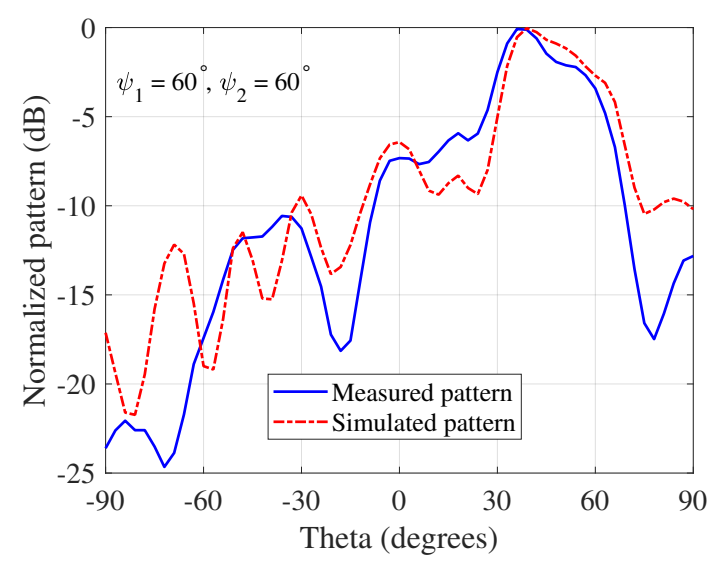

(e)

Figure 16. Simulated and measured elevation plane pattern cuts for the case when lower PGM is fixed $\left(\psi_{1}=0^{\circ}\right)$ and upper PGM is rotated $\left(\psi_{2}\right.$ is varied) from $180^{\circ}$ to $60^{\circ}$ at a step of $30^{\circ}$ : (a) $\psi_{2}=180^{\circ}$, (b) $\psi_{2}=150^{\circ}$, (c) $\psi_{2}=120^{\circ}$ (d) $\psi_{2}=90^{\circ}$ and (e) $\psi_{2}=60^{\circ}$. Each cut is taken at the elevation plane in which the pattern peaks.

$20 \mathrm{GHz}$ ). Spacing in all measurement setups were ensured using Styrofoam layer, which were measured manually and were accurate to about $0.2 \mathrm{~mm}$. The lower PGM was aligned to the $\mathrm{x}$-axis $\left(\psi_{1}=0^{\circ}\right)$, while the upper PGM was physically rotated at steps of $-30^{\circ}$, starting from $180^{\circ}$ to $60^{\circ}$ i.e., $180^{\circ}, 150^{\circ}, 120^{\circ}, 90^{\circ}$ and $60^{\circ}$. Far-field patterns were measured at each step. Elevation plane pattern cuts measured at elevation planes containing the beam peak are compared with the simulated elevation pattern cuts in Fig. 16. There is a reasonable agreement between the predicted and measured radiation pattern results. The 
slight discrepancy can be attributed to the human error involved during the measurement in the chamber.

\section{Discussion}

The beam-steering antennas envisage a critical role in mobile backhaul technology. The electronically scanned antenna systems predominantly rely on active components, are complex and expensive. The NFMS antenna systems are an attractive alternative to traditional lossy beam-steering arrays with nearly equivalent performance with passive components. The absence of active elements such as phase shifters and amplifiers unlocks the potential for use in power-limited mobile environments. NFMS systems made of a base antenna and pair of PGMs use an approach to design both PGMs using cells that have excellent performance for normally incident field, which is not valid for the upper PGM. A Floquet analysis based CMA-ES optimization is implemented to first optimize the lower PGM for normal plane wave incidence and then to optimize upper PGM for oblique plane wave incidence to ensure minimal scattering in unwanted direction because of the coupling between different cells in the PGMs. A compact NFMS designed following the approach indicate ability to steer medium-gain beam to the maximum elevation angle of $\pm 35^{\circ}$ for a $3 \mathrm{~dB}$ reduction in peak directivity. Optimizing the performance of PGMs is a crucial step towards their practical implementation in real-time antenna and RF systems. The proposed optimization approach can also be applied to optimize electrically large PGMs in high-gain NFMS systems. Conventional design and optimization approaches rely on extensive parameter searches and do not account for the near-field interactions that strongly influence the overall system performance. The proposed optimization strategy accounts for the mutual coupling between the neighboring metallic patches.

\section{Conclusion}

A low-cost compact beam-steering system is developed using a pair of truncated PGMs and a medium gain RCA. PGM-pair is optimized using Floquet based analysis and PSO algorithm. Analysis proved that an NFMS system has better steering performance when upper PGM is optimized for oblique incidence, and lower PGM is optimized for normal incidence compared to when both upper and lower PGMs are optimized for normal incidence. The planar profile of the designed steering system facilitates encapsulation into a cylindrical cavity. The metasurfaces are compact and lightweight and can be easily rotated with a pair of low-power stepper motors. Maximum directivity of the antenna system is $17.8 \mathrm{dBi}$, and it can be used to scan any elevation angle up to $35^{\circ}$ for a $3 \mathrm{~dB}$ reduction in peak directivity for all azimuth angles. Beyond $35^{\circ}$ elevation angle, the peak directivity decreases considerably. However, if a directivity as low as $10 \mathrm{dBi}$ is accepted, then the system can be used to scan up to $60^{\circ}$ elevation angle for all azimuth angles. When used as front-end antennas, these steering systems can be controlled remotely to tune the direction in case of any misalignment due to unforeseen circumstances. Since the base antenna is tilt-free and only the upper PGM is rotated, this system does not require any rotary joints.

\section{References}

1. Afzal, M. U., Lalbakhsh, A. \& Esselle, K. P. Compact beam-steered resonant-cavity antenna using near-field phase transformation. In 2019 14th Conference on Industrial and Information Systems (ICIIS), 1-4 (IEEE, 2019).

2. Liaskos, C. et al. Mobility-aware beam steering in metasurface-based programmable wireless environments. In ICASSP 2020-2020 IEEE International Conference on Acoustics, Speech and Signal Processing (ICASSP), 9150-9154 (IEEE, 2020).

3. Artemenko, A. A. \& Maslennikov, R. O. Electronically beam-steerable antenna device (2017). US Patent 9,590,300.

4. Vidal, B., Mengual, T. \& Martí, J. Fast optical beamforming architectures for satellite-based applications. Adv. Opt. Technol. 2012, 1-5 (2012).

5. Stevenson, R., Sazegar, M., Bily, A., Johnson, M. \& Kundtz, N. Metamaterial surface antenna technology: Commercialization through diffractive metamaterials and liquid crystal display manufacturing. In 2016 10th International Congress on Advanced Electromagnetic Materials in Microwaves and Optics (METAMATERIALS), 349-351 (IEEE, 2016).

6. Faenzi, M. et al. Metasurface antennas: new models, applications and realizations. Sci. reports 9, 1-14 (2019).

7. Ostaszewski, M. et al. Risley prism beam pointer. In Free-Space laser communications VI, vol. 6304, 630406 (International Society for Optics and Photonics, 2006).

8. Gagnon, N. \& Petosa, A. Using rotatable planar phase shifting surfaces to steer a high-gain beam. IEEE Transactions on Antennas Propag. 61, 3086-3092 (2013).

9. McEwen, C. \& Khan, M. Beam steering method with improved sidelobe response using dielectric wedges for satellite tv reception. In 1984 14th European Microwave Conference, 681-685 (IEEE, 1984). 
10. Afzal, M. U. \& Esselle, K. P. Steering the beam of medium-to-high gain antennas using near-field phase transformation. IEEE Transactions on Antennas Propag. 65, 1680-1690 (2017).

11. Zhao, X. et al. All-metal beam steering lens antenna for high power microwave applications. IEEE Transactions on Antennas Propag. 65, 7340-7344 (2017).

12. Zhong, Y. C. \& Cheng, Y. J. Generating and steering quasi-non-diffractive beam by near-field planar risley prisms. IEEE Transactions on Antennas Propag. 1-1, DOI: 10.1109/TAP.2020.2998872 (2020).

13. Afzal, M. U. \& Esselle, K. P. Steering the beam of medium-to-high gain antennas using near-field phase transformation. IEEE Transactions on Antennas Propag. 65, 1680-1690 (2017).

14. Wang, J. \& Ramhat-Samii, Y. Phase method: A more precise beam steering model for phase-delay metasurface based risley antenna. In 2019 URSI International Symposium on Electromagnetic Theory (EMTS), 1-4 (2019).

15. Singh, K., Afzal, M. U. \& Esselle, K. P. Designing efficient phase-gradient metasurfaces for near-field meta-steering systems. IEEE Access 9, 109080-109093 (2021).

16. Lalbakhsh, A., Afzal, M. U., Esselle, K. P., Smith, S. L. \& Zeb, B. A. Single-dielectric wideband partially reflecting surface with variable reflection components for realization of a compact high-gain resonant cavity antenna. IEEE Transactions on Antennas Propag. 67, 1916-1921 (2019).

17. Hongnara, T., Chaimool, S., Akkaraekthalin, P. \& Zhao, Y. Design of compact beam-steering antennas using a metasurface formed by uniform square rings. IEEE access 6, 9420-9429 (2018).

18. Achouri, Karim et al. Synthesis of electromagnetic metasurfaces: principles and illustrations. EPJ Appl. Metamaterials 2, 12, DOI: 10.1051/epjam/2015016 (2015).

19. Ali, H., Afzal, M. U., Esselle, K. P. \& Hashmi, R. M. Integration of geometrically different elements to design thin near-field metasurfaces. IEEE Access 8, 225336-225346 (2020).

20. Katare, K. K., Biswas, A. \& Akhtar, M. J. Microwave beam steering of planar antennas by hybrid phase gradient metasurface structure under spherical wave illumination. J. Appl. Phys. 122, 234901 (2017).

21. Singh, K., Afzal, M. U., Kovaleva, M. \& Esselle, K. P. Controlling the most significant grating lobes in two-dimensional beam-steering systems with phase-gradient metasurfaces. IEEE Transactions on Antennas Propag. 68, 1389-1401 (2020).

22. Gagnon, N., Petosa, A. \& McNamara, D. A. Thin phase-correcting lens antennas made using a three-layer phase-shifting surface (pss) at ka band. In 2010 14th International Symposium on Antenna Technology and Applied Electromagnetics the American Electromagnetics Conference, 1-4, DOI: 10.1109/ANTEM.2010.5552469 (2010).

23. Hongnara, T., Chaimool, S., Akkaraekthalin, P. \& Zhao, Y. Design of compact beam-steering antennas using a metasurface formed by uniform square rings. IEEE Access 6, 9420-9429 (2018).

24. Lalbakhsh, A., Afzal, M. U., Esselle, K. P., Smith, S. L. \& Zeb, B. A. Single-dielectric wideband partially reflecting surface with variable reflection components for realization of a compact high-gain resonant cavity antenna. IEEE Transactions on Antennas Propag. 67, 1916-1921 (2019).

25. Hashmi, R. M. \& Esselle, K. P. A class of extremely wideband resonant cavity antennas with large directivity-bandwidth products. IEEE Transactions on Antennas Propag. 64, 830-835 (2015).

26. Wang, J. \& Ramhat-Samii, Y. Phase method: A more precise beam steering model for phase-delay metasurface based risley antenna. In 2019 URSI International Symposium on Electromagnetic Theory (EMTS), 1-4 (IEEE, 2019).

\section{Acknowledgements}

This research was supported by an Australian Research Council Discovery grant and a University of Technology, Faculty of Engineering and IT strategic start-up grant.

\section{Author contributions statement}

Conceptualization: K.S.; methodology: K.S., M.A., and K.E; investigation: all authors; experimental measurements: K.S. and M.U. writing K.S.; funding acquisition, K.E. . All authors reviewed the manuscript.

\section{Competing Interests}

Te authors declare no competing interests. 


\section{Additional information}

Correspondence and requests for materials should be addressed to K.S. Reprints and permissions information is available at www.nature.com/reprints. 